\title{
Estimation of Combining Ability and Gene Action for Various Traits of Baby Corn in Line $\times$ Tester Analysis
}

\author{
Heena Kousar, Sanjeev K. Deshpande* and B. D. Biradar \\ Department of Genetics and Plant Breeding, University of Agricultural Sciences, \\ Dharwad, India \\ *Corresponding author
}

\begin{abstract}
A B S T R A C T
The increasing demand for baby corn intended us towards the development of single cross hybrids for baby corn speciality traits. The experiment consisted of the crossing of nine lines with three testers in line $\times$ tester mating design where the layout of the field was done under RCBD design with two replications at the University of Agricultural Sciences Dharwad during Kharif season 2019. The parental material consists of $\mathrm{F}_{4}$ generation lines belonging to one heterotic group whereas the testers were derived from another heterotic group, representing sufficient genetic diversity and genetic distance between both lines and testers along with two commercial checks HM-4 (National baby corn check) and CPB 468 (Private hybrid). The line BBCP-27-1(L2) was identified as the best general combiner for various baby corn traits such as the number of ears per plant (Prolificacy), cob weight with husk (g), cob weight without husk (g) and baby corn yield without husk per plant (g). The estimates of specific combining effects indicated that the hybrid BBCP-11-1× BBCH-51-2 showed desirable sca effects for cob weight with husk (g), cob weight without husk (g), ear length $(\mathrm{cm})$ and baby corn yield without husk per plant $(\mathrm{g})$. The preponderance of both additive and non-additive gene action was observed for baby corn traits indicating the scope of operation of the selection force.
\end{abstract}

Keywords

Line $\times$ tester, Baby corn, Combining ability, gca, sca

\section{Article Info}

Accepted:

20 October 2020

Available Online:

10 November 2020

\section{Introduction}

Maize being a unique cereal on earth possesses speciality features at the different growth stages, one being the 'Baby corn'. The young unfertilized corn ears harvested 2-3 days after silk emergence depending upon the developmental conditions of the plants is called baby corn. The geographical location of India paves the way towards foreign exchange with many countries belonging to Asia, Europe, Persia and many other Gulf countries. The dehusking of baby corn is mainly hand-operated that demands a huge labour force, suits developing countries like India due to the availability of low-cost labour (Ranjan et al., 2013). The short duration of baby corn helps farmers to take 3-4 crops per year and fits well in different cropping systems by generating additional remuneration to the farmer's income in periurban areas (Dass et al., 2009). The scarcity of work done in the development of high yielding baby corn cultivars leads to their 
limited availability in the market. Currently, some early maturing grain maize cultivars are mainly used for baby corn purposes (Dhasarathan et al., 2012). The line $\times$ tester analysis, is often, used in breeding programs of several cultivated crops, due to its efficiency in selecting parents for the development of hybrids with desired phenotypes. The estimates of general and specific combining ability of parents help breeder in the improvement of various quantitative and qualitative features of the crop and to evolve an economically sound hybrid. The elucidation of gene action also helps in the assessment of the heritability of the trait from one generation to the next generation. Hence, the current study aimed at the identification of promising lines, testers and single cross hybrids with desirable features for baby corn production through estimates of general and specific combining ability along with the type of gene action for various traits of baby corn.

\section{Materials and Methods}

The nine lines and three testers were crossed in $9 \times 3$ (line $\times$ tester) to produce 27 possible $\mathrm{F}_{1}$ hybrids. Nine lines, three testers, $\mathrm{F}_{1}$ hybrids and two commercial check hybrids i.e. HM-4 (National check), CPB 468 (Private check) were grown at the University of Agricultural Sciences Dharwad in India during the 2019 Kharif season. The genotypes were assigned at random to the experimental unit in each block and each row contained 10 plants. Each replication consists of 12 parents (lines and tester), $27 \mathrm{~F}_{1}$ crosses and two check hybrids with a 3-meter long row for each genotype. The distance between rows and plants was $60 \mathrm{~cm}$ and $20 \mathrm{~cm}$ respectively. The twelve lines namely BBCP-8-2, BBCP-27-1, BBCP-37-2, BBCP-49-1, BBCP-27-2, BBCP11-1, ВBCP-41-1, ВBCP-15-3, ВBCP-10-1 and testers $\mathrm{BBCH}-95-2, \mathrm{BBCH}-51-2, \mathrm{BBCH}-$ 120-1 were belonging to two different heterotic groups representing sufficient diversity between lines and testers. The crop was cultivated by following the recommended package of practices for cultivation. The observations were recorded on 10 plants selected by following systematic random sampling. The mean data of the following characters was used for analyzing the general combining ability (GCA), SCA (specific combining ability) and gene action using software WINDOSTAT (V 9.2) following the standard given by Kempthorne (1957). Baby corn yield and component characters are days to $50 \%$ silking, number of baby corn per plant, husked weight of baby corn, dehusked weight of baby corn, baby corn length, baby diameter and baby corn yield without husk per plant.

\section{Results and Discussion}

The analysis of variance showed highly significant differences among the crosses, parents and lines for all the traits. Whereas the testers have shown significance for all the traits except cob weight with husk, ear length and ear diameter as shown in (Table 1). The significant difference of mean squares between parents and crosses for baby corn yield and yield related traits indicated their suitability for combining ability studies as observed by Tucak et al., (2012) and Atif et al., (2012). The significant difference between line $\times$ tester interactions for these traits indicated the presence of specific combining ability attribution in additive and dominance variance for all the baby corn traits. These results coincide with the findings of (Amiruzzaman et al., 2010). The higher value of specific combining ability variance $\left(\sigma^{2} \mathrm{SCA}\right)$ than the general combining ability variance $\left(\sigma^{2} \mathrm{GCA}\right)$, the ratio of $\sigma^{2} \mathrm{GCA} /$ $\sigma^{2} \mathrm{SCA}$ being less than one and degree of dominance being greater than one has a major role in the manifestation of non-additive gene effects (Ojo et al., 2007) as presented in 
(Table 2). In the current study preponderance of non-additive gene action is reported in the expression of various baby corn traits providing vital prospects for the exploitation of gene action in hybrid breeding. In the present study, all the parameters except cob weight without husk have shown higher SCA variances than GCA variances.

The contribution of line $\times$ tester interaction is greater than the contribution of testers for all the traits of baby corn except ear diameter, indicating a higher estimate of specific combining ability effects (Table 3 ). The close examination of parents indicated that none of the parents showed simultaneous significant GCA effects for all the traits studied (Table 4).

The estimation of variations in GCA effects was evaluated among lines and testers for all the traits for the development of promising baby corn hybrids.

Table.1 Analysis of variance for baby corn yield and related traits among $\mathrm{F}_{1}$ hybrids and inbred lines in maize during Kharif 2019

\begin{tabular}{|c|c|c|c|c|c|c|c|c|}
\hline Source of Variation & d.f. & $\begin{array}{l}\text { Days to } \\
50 \% \\
\text { silking }\end{array}$ & $\begin{array}{c}\text { Number of } \\
\text { cobs per } \\
\text { plant }\end{array}$ & $\begin{array}{c}\text { Cob } \\
\text { weight } \\
\text { with husk } \\
\text { (g) }\end{array}$ & $\begin{array}{c}\text { Cob } \\
\text { weight } \\
\text { without } \\
\text { husk (g) }\end{array}$ & $\begin{array}{c}\text { Ear } \\
\text { length } \\
(\mathrm{cm})\end{array}$ & $\begin{array}{c}\text { Ear } \\
\text { diameter } \\
(\mathbf{c m})\end{array}$ & $\begin{array}{c}\text { Baby corn } \\
\text { yield without } \\
\text { husk per plant } \\
\text { (g) }\end{array}$ \\
\hline Replication & 1 & 3.70 & 0.01 & 0.02 & 1.23 & 0.69 & 0.00 & 8.69 \\
\hline Parents & 11 & $8.49 * *$ & $0.83 * *$ & $53.95 * *$ & $6.42 * *$ & $2.37 * *$ & $0.02 * *$ & $78.57 * *$ \\
\hline Females (Lines) & 8 & $7.47 * *$ & $0.77 * *$ & $65.33 * *$ & $7.66^{* *}$ & $2.91 * *$ & $0.03 * *$ & $87.54 * *$ \\
\hline Males (Testers) & 2 & $10.16^{* *}$ & $1.28 * *$ & 0.93 & $4.10 * *$ & 0.51 & 0.01 & $81.91 * *$ \\
\hline $\begin{array}{l}\text { Females V/s Males } \\
\text { (Lines V/s Testers) }\end{array}$ & 1 & $13.34 * *$ & 0.39 & $68.93 * *$ & 1.14 & 1.78 & 0.00 & 0.13 \\
\hline Crosses & 26 & $6.89 * *$ & $0.98 * *$ & $54.27 * *$ & $6.40 * *$ & $3.80 * *$ & $0.01 *$ & $150.98 * *$ \\
\hline Parents V/s Crosses & 1 & 0.92 & $3.18 * *$ & $3036.52 * *$ & $72.39 * *$ & $29.38 * *$ & $0.50 * *$ & $1492.58 * *$ \\
\hline Error & 38 & 1.38 & 0.10 & 2.49 & 0.49 & 0.77 & 0.00 & 10.83 \\
\hline
\end{tabular}

* - Significant at $5 \%$ level of significance ** - Significant at $1 \%$ level of significance

Table.2 Estimates of GCA variance, SCA variance and predictability ratio $\left(\sigma^{2} \mathrm{GCA} / \sigma^{2} \mathrm{SCA}\right)$ along with prevailing gene action

\begin{tabular}{|l|l|c|c|c|c|c|c|}
\hline SI. No. & Characters & $\boldsymbol{\sigma}^{\mathbf{2}} \mathbf{G C A}$ & $\boldsymbol{\sigma}^{\mathbf{2}} \mathbf{S C A}$ & $\boldsymbol{\sigma}^{\mathbf{2}} \mathbf{G C A} / \boldsymbol{\sigma}^{\mathbf{2}} \mathbf{S C A}$ & $\boldsymbol{\sigma}^{\mathbf{2}} \mathbf{A}$ & $\boldsymbol{\sigma}^{\mathbf{2}} \mathbf{D}$ & $\boldsymbol{\sigma}^{\mathbf{2}} \mathbf{A} / \boldsymbol{\sigma}^{\mathbf{2}} \mathbf{D}$ \\
\hline $\mathbf{1 .}$ & Days to 50 \% silking & 0.18 & 3.26 & 0.05 & 0.37 & 3.26 & 0.11 \\
\hline $\mathbf{2 .}$ & Number of cobs per plant & 0.10 & 0.32 & 0.31 & 0.20 & 0.32 & 0.62 \\
\hline $\mathbf{3 .}$ & Cob weight with husk & 1.70 & 33.38 & 0.05 & 3.41 & 33.38 & 0.10 \\
\hline $\mathbf{4 .}$ & Cob weight without husk & 0.42 & 2.64 & 0.15 & 0.85 & 2.64 & 0.32 \\
\hline $\mathbf{5 .}$ & Ear length & 0.38 & 1.36 & 0.27 & 0.76 & 1.36 & 0.55 \\
\hline $\mathbf{6 .}$ & Ear diameter & 0.00 & 0.00 & 0.00 & 0.00 & 0.00 & 0.00 \\
\hline $\mathbf{7 .}$ & $\begin{array}{l}\text { Baby corn yield without husk } \\
\text { per plant }\end{array}$ & 13.02 & 59.84 & 0.21 & 26.05 & 59.84 & 0.43 \\
\hline
\end{tabular}


Table.3 The proportion of contribution of female, male and female $\times$ male for baby corn yield and related traits

\begin{tabular}{|c|c|c|c|c|}
\hline $\begin{array}{l}\text { SI. } \\
\text { No. }\end{array}$ & Characters & $\begin{array}{l}\text { Contribution of the } \\
\text { line }(\%)\end{array}$ & $\begin{array}{l}\text { Contribution of the } \\
\text { tester }(\%)\end{array}$ & $\begin{array}{l}\text { Contribution of } \\
\text { line } \times \text { tester }(\%)\end{array}$ \\
\hline 1. & Days to $50 \%$ silking & 28.81 & 0.80 & 70.38 \\
\hline 2. & Number of cobs per plant & 40.21 & 11.05 & 48.73 \\
\hline 3. & Cob weight with husk (g) & 21.67 & 0.83 & 77.48 \\
\hline 4. & Cob weight without husk ( $\mathrm{g}$ ) & 38.69 & 4.20 & 57.09 \\
\hline 5. & Ear length $(\mathrm{cm})$ & 40.69 & 9.65 & 49.64 \\
\hline 6. & Ear diameter $(\mathrm{cm})$ & 12.97 & 21.03 & 65.99 \\
\hline 7. & $\begin{array}{l}\text { Baby corn yield without } \\
\text { husk per plant }(\mathrm{g})\end{array}$ & 38.01 & 7.78 & 54.20 \\
\hline
\end{tabular}

Table.4 General combining ability ( $g c a$ ) effects of inbred lines for baby corn yield and related traits in maize

\begin{tabular}{|c|c|c|c|c|c|c|c|c|}
\hline \multirow[b]{2}{*}{$\begin{array}{l}\text { SI. } \\
\text { No. }\end{array}$} & \multirow[b]{2}{*}{ Inbreds } & \multicolumn{7}{|c|}{ Characters } \\
\hline & & $\begin{array}{c}\text { Days to } \\
50 \% \\
\text { silking }\end{array}$ & $\begin{array}{l}\text { Number } \\
\text { of cobs } \\
\text { per plant }\end{array}$ & $\begin{array}{c}\text { Cob } \\
\text { weight } \\
\text { with husk } \\
\text { (g) }\end{array}$ & $\begin{array}{c}\text { Cob weight } \\
\text { without } \\
\text { husk (g) }\end{array}$ & $\begin{array}{c}\text { Ear } \\
\text { length } \\
(\mathbf{c m})\end{array}$ & $\begin{array}{c}\text { Ear } \\
\text { diameter } \\
(\mathbf{c m})\end{array}$ & $\begin{array}{c}\text { Baby } \\
\text { corn yield } \\
\text { without } \\
\text { husk per } \\
\text { plant (g) }\end{array}$ \\
\hline \multicolumn{9}{|c|}{ Females (Lines) } \\
\hline 1. & ВВСР-8-2 & $-1.88 * *$ & -0.18 & 0.30 & $1.68 * *$ & $1.11 * *$ & 0.01 & $3.52 *$ \\
\hline 2. & ВВСР-27-1 & -0.88 & $0.76 * *$ & $1.07 *$ & $1.14 * *$ & 0.33 & 0.02 & $9.46 * *$ \\
\hline 3. & ВВСР-37-2 & 0.44 & $-0.48 * *$ & $-1.44 * *$ & $0.83 *$ & $1.02 * *$ & 0.02 & -0.95 \\
\hline 4. & ВВСР-49-1 & -0.55 & $-0.30 *$ & $-4.77 * *$ & $-1.75 * *$ & $-1.61 * *$ & -0.00 & $-6.63 * *$ \\
\hline 5. & ВBCP-27-2 & 0.11 & $0.70 * *$ & $3.36 * *$ & 0.66 & $0.56 *$ & 0.03 & $7.11 * *$ \\
\hline 6. & BBCP-11-1 & 0.11 & 0.08 & -0.52 & $-1.36 * *$ & -0.46 & -0.06 & $-3.35 *$ \\
\hline 7. & ВBCP-41-1 & -0.05 & -0.03 & $-2.11 * *$ & -0.34 & -0.04 & 0.01 & -1.28 \\
\hline 8. & ВBCP-15-3 & $1.44 * *$ & $-0.51 * *$ & $2.38 * *$ & -0.52 & $-1.06 * *$ & -0.04 & $-5.82 * *$ \\
\hline 9. & ВBCP-10-1 & $1.27 *$ & -0.03 & $1.73 * *$ & -0.34 & 0.14 & 0.00 & -2.04 \\
\hline \multicolumn{9}{|c|}{ Male (Testers) } \\
\hline 1. & BBCH-95-2 & -0.05 & $-0.32 * *$ & 0.05 & -0.22 & -0.11 & $0.04 *$ & $-3.11 * *$ \\
\hline 2. & BBCH-51-2 & 0.22 & $0.18 *$ & -0.59 & -0.28 & $-0.44 * *$ & $-0.05 *$ & 0.45 \\
\hline 3. & ВBCH-120-1 & -0.16 & 0.13 & 0.54 & $0.50 *$ & $0.56 * *$ & 0.00 & $2.65 * *$ \\
\hline
\end{tabular}

\footnotetext{
$*$ - Significant at $5 \%$ level of significance $* *$ - Significant at $1 \%$ level of significance
} 
Table.5 Specific combining ability $\left(s c a\right.$ ) effects of $\mathrm{F}_{1}$ hybrids for baby corn yield and related traits

\begin{tabular}{|c|c|c|c|c|c|c|c|c|}
\hline \multirow[b]{2}{*}{$\begin{array}{l}\text { SI. } \\
\text { No. }\end{array}$} & \multirow[b]{2}{*}{ Hybrids } & \multicolumn{7}{|c|}{ Characters } \\
\hline & & $\begin{array}{c}\text { Days to } \\
50 \% \\
\text { silking }\end{array}$ & $\begin{array}{c}\text { Number } \\
\text { of cobs } \\
\text { per } \\
\text { plant }\end{array}$ & $\begin{array}{c}\text { Cob } \\
\text { weight } \\
\text { with } \\
\text { husk (g) }\end{array}$ & $\begin{array}{c}\text { Cob } \\
\text { weight } \\
\text { without } \\
\text { husk (g) }\end{array}$ & $\begin{array}{c}\text { Ear } \\
\text { length } \\
(\mathrm{cm})\end{array}$ & $\begin{array}{c}\text { Ear } \\
\text { diame } \\
\text { ter } \\
(\mathrm{cm})\end{array}$ & $\begin{array}{l}\text { Baby corn } \\
\text { yield without } \\
\text { husk per } \\
\text { plant (g) }\end{array}$ \\
\hline 1. & BBCP-8-2 × BBCH-95-2 & 0.22 & 0.15 & 0.06 & 0.48 & $0.99 *$ & 0.07 & 2.48 \\
\hline 2. & ВВСР-8-2 $\times$ ВВCH-51-2 & -0.05 & -0.15 & $3.75 * *$ & 0.51 & 0.52 & -0.02 & 0.58 \\
\hline 3. & ВВСР-8-2 $\times$ ВBCH-120-2 & -0.16 & -0.00 & $-3.81 * *$ & -0.99 & $-1.51^{* *}$ & -0.05 & -3.06 \\
\hline 4. & ВВСР-27-1 × BBCH-95-2 & $1.72 *$ & $-0.79 * *$ & $-6.13 * *$ & 0.42 & 0.06 & 0.02 & -5.26 \\
\hline 5. & BBCP-27-1 $\times$ BBCH-51-2 & $-2.05 *$ & $1.05 * *$ & 0.18 & -0.01 & 0.31 & -0.01 & $8.29 * *$ \\
\hline 6. & ВВСР-27-1 × ВВСН-120-2 & 0.33 & -0.25 & $5.94 * *$ & -0.41 & -0.37 & -0.01 & -3.03 \\
\hline 7. & ВВСР-37-2 × ВBCH-95-2 & 0.38 & 0.45 & $8.20 * *$ & $1.66 * *$ & 0.48 & 0.07 & $7.95 * *$ \\
\hline 8. & ВВСР-37-2 $\times$ ВBCH-51-2 & 1.11 & 0.25 & $-9.23 * *$ & 0.84 & -0.07 & 0.06 & 4.27 \\
\hline 9. & ВВСР-37-2 $\times$ ВВСН-120-2 & -1.5 & $-0.70 * *$ & 1.02 & $-2.50 * *$ & -0.40 & -0.13 & $-12.22 * *$ \\
\hline 10. & ВВСР-49-1 × ВBCH-95-2 & 0.88 & -0.12 & 0.23 & 0.29 & 0.77 & 0.07 & -0.36 \\
\hline 11. & BBCP-49-2 × BBCH-51-2 & -0.88 & $-0.93 * *$ & $2.56 * *$ & $-1.66 * *$ & $-1.94^{* *}$ & -0.12 & $-10.22 * *$ \\
\hline 12. & ВВСР-49-3 × ВBCH-120-2 & 0.00 & $1.06 * *$ & $-2.80 * *$ & $1.37 *$ & $1.17 * *$ & 0.05 & $10.59 * *$ \\
\hline 13. & BBCP-27-2 × BBCH-95-2 & $-2.77 * *$ & -0.07 & $5.03 * *$ & 1.09 & 0.14 & 0.07 & 3.24 \\
\hline 14. & ВBCP-27-3 × BBCH-51-2 & $1.94 *$ & -0.23 & $-5.95 * *$ & -0.73 & 15 & -0.03 & -4.85 \\
\hline 15. & BВCP-27-4 × BBCH-120-2 & 0.83 & 0.31 & 0.91 & -0.35 & -0.29 & -0.03 & 1.61 \\
\hline 16. & BBCP-11-1 × BBCH-95-2 & 1.22 & -0.41 & $-8.93 * *$ & $-2.75 * *$ & $-2.29 * *$ & -0.12 & $-10.28 * *$ \\
\hline 17. & BBCP-11-2 × BBCH-51-2 & 0.44 & 0.38 & $3.92 * *$ & $2.48 * *$ & $1.35 * *$ & 0.12 & $10.39 * *$ \\
\hline 18. & ВВСР-11-3 × ВBCH-120-2 & -1.66 & 0.02 & $5.00 * *$ & 0.27 & $0.94 *$ & -0.00 & -0.10 \\
\hline 19. & BBCP-41-1 × BBCH-95-2 & 0.88 & 0.10 & $4.05 * *$ & 0.44 & 0.77 & -0.01 & 1.94 \\
\hline 20. & BBCP-41-1 × BBCH-51-2 & $-1.88 *$ & -0.4 & $-3.44 * *$ & $-2.18 * *$ & $-1.18^{* *}$ & -0.05 & $-10.11 * *$ \\
\hline 21. & ВBCP-41-1 × BBCH-120-2 & 1.00 & 0.29 & -0.61 & $1.73 * *$ & 0.41 & 0.06 & $8.17 * *$ \\
\hline 22. & ВBCP-15-3 × BBCH-95-2 & 1.38 & $0.68 *$ & $-3.23 * *$ & -1.05 & $-1.49 * *$ & -0.05 & 1.59 \\
\hline 23. & BBCP-15-3 × BBCH-51-2 & 0.11 & -0.11 & $5.77 * *$ & $1.65 * *$ & $1.23 * *$ & 0.01 & 4.00 \\
\hline 24. & ВBCP-15-3 × BBCH-120-2 & -1.50 & $-0.57 *$ & $-2.53 * *$ & -0.59 & 0.26 & 0.03 & $-5.60 *$ \\
\hline 25. & BBCP-10-1 × BBCH-95-2 & $-3.94 * *$ & 0.00 & 0.71 & -0.57 & 0.55 & -0.12 & -1.30 \\
\hline 26. & ВВCP-10-1 × BBCH-51-2 & 1.27 & 0.15 & $2.42 *$ & -0.89 & -0.36 & 0.04 & -2.35 \\
\hline 27. & BВCP-10-1 × BBCH-120-2 & $2.66 * *$ & -0.15 & $-3.13 * *$ & $1.47 *$ & -0.18 & 0.08 & 3.65 \\
\hline
\end{tabular}

\footnotetext{
* - Significant at 5\% level of significance ** - Significant at $1 \%$ level of significance
} 
Table.6 Best parents identified on basis of GCA effects and per se performance for different baby corn traits

\begin{tabular}{|c|c|c|c|}
\hline Characters & Parent & GCA effects & Per se performance \\
\hline Days to $50 \%$ silking & BBCP-8-2 & $-1.88 * *$ & 58.50 \\
\hline \multirow[t]{3}{*}{ Number of cobs per plant } & BBCP-27-1 & $0.76 * *$ & 3.10 \\
\hline & BBCP-27-2 & $0.70 * *$ & 3.75 \\
\hline & BBCH-51-2 & $0.18 *$ & 2.30 \\
\hline \multirow[t]{4}{*}{ Cob weight with husk (g) } & BBCP-27-2 & $3.36 * *$ & 22.15 \\
\hline & ВBCР-15-3 & $2.38 * *$ & 39.38 \\
\hline & ВBCP-10-1 & $1.73 * *$ & 23.38 \\
\hline & ВBCP-27-1 & $1.07 *$ & 26.32 \\
\hline \multirow[t]{4}{*}{ Cob weight without husk (g) } & ВBCP-8-2 & $1.68 * *$ & 4.16 \\
\hline & ВBCP-27-1 & $1.14 * *$ & 6.18 \\
\hline & BBCP-37-2 & $0.83 *$ & 2.92 \\
\hline & BBCH-120-1 & $0.50 *$ & 4.42 \\
\hline \multirow[t]{4}{*}{ Ear length (cm) } & BBCP-8-2 & $1.11 * *$ & 7.37 \\
\hline & ВBCР-37-2 & $1.02 * *$ & 6.64 \\
\hline & ВBCР-27-2 & $0.56 *$ & 6.75 \\
\hline & BBCH-120-1 & $0.56 * *$ & 7.03 \\
\hline Ear diameter (cm) & BBCH-95-2 & $0.04 *$ & 1.08 \\
\hline \multirow{4}{*}{$\begin{array}{l}\text { Baby corn yield without husk per } \\
\text { plant }(\mathrm{g})\end{array}$} & ВBCР-27-1 & $9.46 * *$ & 19.25 \\
\hline & ВВСР-27-2 & $7.11 * *$ & 17.82 \\
\hline & ВВСР-8-2 & $3.52 *$ & 13.29 \\
\hline & ВBCH-120-1 & $2.65 * *$ & 17.30 \\
\hline
\end{tabular}

* - Significant at 5\% level of significance ** - Significant at $1 \%$ level of significance 
Table.7 Top three single cross hybrids for baby corn yield and yield related traits on per se mean

\begin{tabular}{|c|c|c|c|c|c|}
\hline $\begin{array}{l}\text { Sl. } \\
\text { No. }\end{array}$ & Characters & Cross & $\begin{array}{l}\text { Per se } \\
\text { mean }\end{array}$ & sca effect & $\begin{array}{l}\text { gca effect } \\
\text { of inbreds }\end{array}$ \\
\hline \multirow[t]{3}{*}{1.} & Days to $50 \%$ silking & ВBCP-27-2 $\times$ ВBCH-95-2 & 53.5 & $-2.77 * *$ & $\mathrm{M} \times \mathrm{M}$ \\
\hline & & ВBCP-27-1 $\times$ ВBCH-51-2 & 53.5 & $-2.05 *$ & $\mathrm{M} \times \mathrm{M}$ \\
\hline & & BВCP-10-1 × BBCH-95-2 & 53.5 & $-3.94 * *$ & $\mathrm{~L} \times \mathrm{M}$ \\
\hline \multirow[t]{3}{*}{2.} & $\begin{array}{l}\text { Number of cobs per } \\
\text { plant }\end{array}$ & ВВСР-27-1 $\times$ ВBCH $-51-2$ & 5.35 & $1.05 * *$ & $\mathrm{H} \times \mathrm{H}$ \\
\hline & & BBCP-27-2 × BBCH-120-1 & 4.5 & 0.31 & $\mathrm{H} \times \mathrm{M}$ \\
\hline & & BBCP-49-1 × BBCH-120-1 & 4.25 & $1.06 * *$ & $\mathrm{~L} \times \mathrm{M}$ \\
\hline \multirow[t]{3}{*}{3.} & $\begin{array}{l}\text { Cob weight with husk } \\
\text { (g) }\end{array}$ & ВBCP-27-2 × ВBCH-95-2 & 47.94 & $5.03 * *$ & $\mathrm{H} \times \mathrm{M}$ \\
\hline & & BBCP-27-1 × BBCH-120-1 & 47.05 & $5.94 * *$ & $\mathrm{H} \times \mathrm{M}$ \\
\hline & & ВBCP-15-3 × ВBCH-51-2 & 47.05 & $5.77 * *$ & $\mathrm{H} \times \mathrm{M}$ \\
\hline \multirow[t]{3}{*}{4.} & $\begin{array}{l}\text { Cob weight without } \\
\text { husk }(\mathrm{g})\end{array}$ & ВВСР-37-2 × ВBCH-95-2 & 9.33 & $1.66 * *$ & $\mathrm{H} \times \mathrm{M}$ \\
\hline & & BBCP-8-2 $\times$ BBCH-95-2 & 9 & 0.48 & $\mathrm{H} \times \mathrm{M}$ \\
\hline & & BBCP-8-2 × BBCH-51-2 & 8.96 & 0.51 & $\mathrm{H} \times \mathrm{M}$ \\
\hline \multirow[t]{3}{*}{5.} & Ear length $(\mathrm{cm})$ & BBCP-8-2 × BBCH-95-2 & 10.18 & $0.99 *$ & $\mathrm{H} \times \mathrm{M}$ \\
\hline & & ВBCP-37-2 × ВBCH-95-2 & 9.58 & 0.48 & $\mathrm{H} \times \mathrm{M}$ \\
\hline & & ВBCP-8-2 × ВBCH-51-2 & 9.37 & 0.52 & $\mathrm{H} \times \mathrm{L}$ \\
\hline \multirow[t]{3}{*}{6.} & Ear diameter $(\mathrm{cm})$ & BВCP-27-2 $\times$ ВBCH-95-2 & 1.38 & 0.07 & $\mathrm{M} \times \mathrm{H}$ \\
\hline & & ВBCP-37-2 × ВBCH-95-2 & 1.37 & 0.07 & $\mathrm{M} \times \mathrm{H}$ \\
\hline & & BВCP-8-2 × ВBCH-95-2 & 1.37 & 0.07 & $\mathrm{M} \times \mathrm{H}$ \\
\hline \multirow[t]{3}{*}{7.} & $\begin{array}{l}\text { Baby corn yield without } \\
\text { husk per plant (g) }\end{array}$ & BBCP-27-1 × BBCH-51-2 & 42.32 & $8.29 * *$ & $\mathrm{H} \times \mathrm{M}$ \\
\hline & & ВВСР-27-2 × ВBCH-120-1 & 35.5 & 1.61 & $\mathrm{H} \times \mathrm{H}$ \\
\hline & & BBCP-41-1 × BBCH-120-1 & 33.65 & $8.17 * *$ & $\mathrm{M} \times \mathrm{H}$ \\
\hline
\end{tabular}

$\mathrm{H}$ - Significant $g c a$ in the desirable direction

M- Non- significant $\mathrm{gca}$

L- Significant $g c a$ in an undesirable direction

* - Significant at $5 \%$ level of significance ** - Significant at $1 \%$ level of significance

Among the lines, BBCP-8-2, BBCP-27-1 and BBCP-27-2 had favorable genes for baby corn yield without husk per plant due to significant GCA effects. Generally, the higher per se performance for all the traits was associated with higher GCA effects except for days to $50 \%$ silking. The line BBCP-8-2 has shown topmost performance for days to $50 \%$ silking and identified as the desirable parent for early maturity. The results obtained confirm with the investigation of Ranjith et al., (2014) and Kumari et al., (2017).

The lines BBCP-27-1, BBCP-27-2 and BBCP-8-2 were observed to have the desired direction for maximum traits where the line BBCP-27-1 has shown significant GCA effects for the number of cobs per plant, cob weight with husk, cob weight without husk and baby corn yield without husk per plant. 
Whereas, the line BBCP-27-2 has shown significant GCA effects for traits such as the number of cobs per plant, cob weight with husk, ear length and baby corn yield without husk per plant. Likewise, the line BBCP-8-2 has resulted in desirable GCA effects for days to $50 \%$ silking, cob weight without husk, ear length and baby corn yield without husk per plant. The tester BBCH-120-1 was identified as the best combiner with high GCA effects for cob weight without husk, ear length and baby corn yield without husk per plant similar to Dhasarthan et al., (2012). Whereas, the testers BBCH-51-2 and BBCH-95-2 have shown high GCA effects for the number of ears per plant and ear diameter respectively. The estimates of SCA effects indicated that none of the cross combinations exhibited desirable significant effects for all the traits studied (Table 5). Since baby corn yield is the ultimate goal of the baby corn breeding program. For this trait five hybrid combinations viz. BBCP-27-1 × BBCH-51-2, BBCP-37-2 $\times$ BBCH-95-2, BBCP-49-1 $\times$ BBCH-120-1, BBCP-11-1 × BBCP-51-2 and BBCP-41-1 $\times$ BBCH-120-1 were found good specific combinations based on high and significant SCA effects similar to the findings of Kumari et al., (2017) and Izhar and Chakraborty (2013). Two cross combinations were reported to be the best combinations for various baby corn traits among which the hybrid BBCP-49-1 × BBCH-120-1 has shown good specific combining ability for the number of cobs per plant, cob weight without husk, baby corn length and baby corn yield without husk per plant. Similarly, for many other traits, a set of good specific combinations were identified based on high mean performance and SCA effects. The best hybrid combinations for various baby corn traits are represented in (Table 6). Hence (Table 7) includes the top-ranking cross combinations based on per se mean and SCA effects of crosses for various traits of baby corn.
In conclusion the out of 12 parental inbred lines, the line BBCP-27-1 was identified as the best general combiner for the majority of baby corn traits by exhibiting desirable GCA effects. Hence, the line can be extensively used in baby corn breeding programs. The hybrid BBCP-49-1 $\times$ BBCH-120-1 was identified as a good combiner for baby corn yield without husk per plant and the number of cobs per plant. These promising genotypes can be further utilized in advanced breeding strategies for baby corn yield and quality improvements.

\section{Acknowledgments}

The authors are highly thankful to the College of Agriculture, Dharwad (UASD), Karnataka and IARI-RRC, Dharwad, Karnataka for providing all assistance and research material.

\section{References}

Amiruzzaman, M., M. Islam, L. Hassan and Rohman, M. 2010. Combining ability and heterosis for yield and component characters in maize. Academic Journal of Plant Sciences. 3(2): 79-84.

Atif, I. A., and Awadalla, M. M. 2012. Combining ability and heterosis for yield and yield components in maize (Zea mays L.). Australian Journal of Basic and Applied Sciences. 6(10): 3641.

Dass, S., A. Kwatra, D. Pal, V. K. Yadav, R. Sujay and Singh R. P. 2007. Baby corn in India. Directorate of Maize Research, Pusa Campus, New Delhi. Pp. 1-2.

Dhasarathan, M., C. Babu, K. Iyanar and Velayudham K. 2012. Studies on genetic potential of baby corn (Zea mays L.) hybrids for yield and quality traits. Electronic Journal of Plant Breeding. 3: 853-860.

Izhar, T., and Chakraborty, M. 2013. Genetic analysis of yield and quality parameters 
in baby corn (Zea mays L.). Current Advances in Agricultural Sciences. 5(1): 27-31.

Kempthorne, O., 1957. An Introduction to Genetic Statistics. New York: John Wiley \& Sons, Inc. London, Chapman \& Hall Ltd. Pp. 458-471.

Kumari, H., N. Kumar, K. M. Nikhil, R. B. P. Nirala and Mishra A. 2017. Studies on Baby Corn (Zea mays L.) genotypes for their combining ability and heterosis based on pre and post-harvest traits. Bulletin of Environment, Pharmacology and Life Sciences. 6(1): 486-491.

Ojo, G. O., D. K. Adedzwa and Bello L. L. 2007. Combining ability estimates and heterosis for grain yield and yield components in maize (Zea mays L.). Journal of Sustainable Development in Agriculture \& Environment. 3: 49-57.
Ranjan, J. K., N. Ahmed, B. Das, P. Ranjan and Mishra B. 2013. Green technology for production of baby corn (Zea mays L.) under northwest Himalayan conditions. International Journal of ChemTech Research. 29: 71-77.

Rajitha, A., B. D. Ratna, A. M. Lal and Rao S. 2014. Heterosis and combining ability for grain yield and yield component traits in maize (Zea mays L.). Electronic Journal of Plant Breeding. 5(3): 378-384.

Tucak, M., S. Popovic, T. Cupic, V. Spanic, B. Simic and Meglic V. 2012. Combining abilities and heterosis for dry matter yield in alfalfa diallel crosses. NARDI FUNDULEA. Romanian Agricultural Research. 29: 71-77.

\section{How to cite this article:}

Heena Kousar, Sanjeev K. Deshpande and Biradar, B. D. 2020. Estimation of Combining Ability and Gene Action for Various Traits of Baby Corn in Line $\times$ Tester Analysis. Int.J.Curr.Microbiol.App.Sci. 9(11): 2799-2807. doi: https://doi.org/10.20546/ijcmas.2020.911.339 